\title{
A personalized approach to the acute respiratory distress syndrome: recent advances and future challenges
}

\author{
Elena Spinelli ${ }^{1}$, Domenico L. Grieco ${ }^{2}$, Tommaso Mauri ${ }^{1}$ \\ ${ }^{1}$ Department of Anesthesia, Critical Care and Emergency, IRCCS Foundation "Ca' Granda" "Maggiore Policlinico" Hospital, Milan, Italy; \\ ${ }^{2}$ Department of Anesthesiology and Intensive Care Medicine, Catholic University of the Sacred Heart, Fondazione Policlinico Universitario A. \\ Gemelli IRCCS, Rome, Italy \\ Correspondence to: Tommaso Mauri. Department of Anesthesia, Critical Care and Emergency, IRCCS Foundation “Ca' Granda” "Maggiore \\ Policlinico” Hospital, Via F. Sforza 35, 20122 Milan, Italy. Email: tommaso.mauri@unimi.it. \\ Provenance: This is an invited article commissioned by the Section Editor Zhiheng Xu (State Key Laboratory of Respiratory Disease, Guangzhou \\ Institute of Respiratory Disease, Department of Intensive Care, The First Affiliated Hospital of Guangzhou Medical University, Guangzhou, China). \\ Comment on: Constantin JM, Jabaudon M, Lefrant JY, et al. Personalised mechanical ventilation tailored to lung morphology versus low positive \\ end-expiratory pressure for patients with acute respiratory distress syndrome in France (the LIVE study): a multicentre, single-blind, randomised \\ controlled trial. Lancet Respir Med 2019;7:870-80.
}

Submitted Oct 22, 2019. Accepted for publication Nov 24, 2019.

doi: $10.21037 /$ jtd.2019.11.61

View this article at: http://dx.doi.org/10.21037/jtd.2019.11.61

\section{Introduction}

Since its very first description, understanding the acute respiratory distress syndrome (ARDS) has been a major research and clinical challenge due to heterogeneity in causes, manifestations, physiologic derangements and response to treatment. Recently, growing evidences on the mechanisms underlying ARDS pathophysiology and randomized trials yielding negative results generated a worldwide collaborative effort to define more homogenous subgroups of ARDS subjects and titrate personalized interventions. A recently published trial (the LIVE study) tried to maximize this effort and compared for the first time personalized $v s$. conventional protective ventilation strategies (1). In the present article, we describe how ventilation in ARDS could be personalized based on sound pathophysiological subgroups and comment the results from the LIVE study.

\section{Personalized classification of ARDS}

The ideal definition of ARDS should reach a compromise between facilitating the recognition of this syndrome at the bedside and differentiating subgroups of patients with different biology, respiratory physiology, response to treatment and outcome. According to the Berlin definition (2), stratification of ARDS is based on the degree of oxygenation impairment assessed by the ratio of $\mathrm{PaO}_{2}$ to $\mathrm{FiO}_{2}(\mathrm{P} / \mathrm{F})$. The Berlin definition doesn't recommend standardized ventilator settings, although positive endexpiratory pressure (PEEP) and $\mathrm{FiO}_{2}$ affect the $\mathrm{P} / \mathrm{F}$. Moreover, it disregards other indexes of severity known to describe ARDS pathophysiology and predict response to treatment, such as dead space, lung elastance, recruitability and inhomogeneity (3). Although clinical studies showed that, on average, all these indexes progressively increase from mild to moderate to severe subjects according to the Berlin definition, large variability exists within each subgroup. Therefore, the stratification proposed by the current definition may work for increasing patient homogeneity in clinical studies on personalized treatments (i.e., use of more invasive and risky maneuvers in more severe subjects), but it is largely inadequate to define the patient-specific pathophysiology and to tailor treatment on individual requirements.

In the perspective of protective mechanical ventilation, personalized treatment means to adjust ventilator settings to minimize the risk of ventilator-induced lung injury (VILI) in the individual patient, rather than applying average population-based treatments. While clinical trials demonstrated the benefits of limiting tidal volume 
Table 1 Summary of personalized ARDS classification and ventilator settings

\begin{tabular}{|c|c|c|c|}
\hline $\begin{array}{l}\text { Criteria for ARDS } \\
\text { classification }\end{array}$ & Sub-phenotype & Tidal volume & PEEP \\
\hline \multirow[t]{2}{*}{ Recruitability } & Higher & 5-6 mL/kg PBW and DP $<14 \mathrm{cmH}_{2} \mathrm{O}$ & $\begin{array}{l}\text { Higher by assessing recruitment and over-distension } \\
\text { by bedside method (EIT, EELV measure, mechanics) }\end{array}$ \\
\hline & Lower & 6-8 mL/kg PBW and DP $<14 \mathrm{cmH}_{2} \mathrm{O}$ & Lower PEEP/FiO ${ }_{2}$ table \\
\hline \multirow[t]{2}{*}{ Oxygenation } & $P / F \leq 150$ & 5-6 mL/kg PBW and DP $<14 \mathrm{cmH}_{2} \mathrm{O}$ & $\begin{array}{l}\text { Higher by increasing Pplat to } 28-30 \mathrm{cmH}_{2} \mathrm{O} \text { or } \\
\text { elastance-derived } \mathrm{P}_{\mathrm{L}} \text { to } 20-22 \mathrm{cmH}_{2} \mathrm{O}\end{array}$ \\
\hline & $\mathrm{P} / \mathrm{F}>150$ & 6-8 mL/kg PBW and DP $<14 \mathrm{cmH}_{2} \mathrm{O}$ & Lower PEEP/FiO ${ }_{2}$ table \\
\hline \multirow[t]{2}{*}{ Lung morphology } & Non-focal & 5-6 mL/kg PBW and DP $<14 \mathrm{cmH}_{2} \mathrm{O}$ & $\begin{array}{l}\text { Higher by assessing recruitment and over-distension } \\
\text { by bedside method (EIT, EELV measure, mechanics) }\end{array}$ \\
\hline & Focal & 6-8 mL/kg PBW and DP $<14 \mathrm{cmH}_{2} \mathrm{O}$ & Lower PEEP/FiO ${ }_{2}$ table \\
\hline
\end{tabular}

ARDS, acute respiratory distress syndrome; PEEP, positive end expiratory pressure; PBW, predicted body weight; DP, driving pressure; EIT, electrical impedance tomography; EELV, end-expiratory lung volume; $\mathrm{P} / \mathrm{F}, \mathrm{PaO}_{2} / \mathrm{FiO}_{2}$ ratio; Pplat, inspiratory plateau pressure; $\mathrm{P}_{\mathrm{L}}$, transpulmonary pressure.

and plateau pressure (4), increasing understanding of the heterogeneous pathophysiology of ARDS opened the way for individualized ventilator settings based on respiratory mechanics, as a sound alternative to arbitrary universal targets (5). The description of the "baby lung" concept supported the idea that tidal volume $\left(\mathrm{V}_{\mathrm{T}}\right)$ needs to be scaled to the functional lung size (to decrease lung "strain"), while appreciation of the variability in lung and chest wall respective contribution to respiratory system elastance shifted the focus from airway pressure to transpulmonary pressure (to limit lung "stress") $(6,7)$. Lung recruitability and inhomogeneity were also shown to influence the physiological and clinical effect of ventilator settings. Since strain, stress, recruitability and inhomogeneity are not routinely assessed at the bedside, attempts were done to identify clinical sub-phenotypes with more uniform pathophysiology.

The distinction between pulmonary and extra-pulmonary ARDS was proposed based on the direct or indirect etiology of lung injury. Extra-pulmonary ARDS was initially thought to be characterized by higher chest wall elastance and higher recruitability, but this was not confirmed by subsequent studies (8). Indeed, recent data indicate that recruitability is related to the severity rather than to the origin of ARDS but is highly inter-individually variable (3).

To further analyze the value of impaired oxygenation in dissecting ARDS sub-phenotypes, a P/F threshold of
$150 \mathrm{mmHg}$ measured at standardized PEEP level of $5 \mathrm{cmH}_{2} \mathrm{O}$ was suggested to identify two populations with more homogenous anatomical and physiological characteristics (9): the mild-moderate and the moderatesevere subjects.

Lung morphology assessed by radiological images is another proposed criterion for clinical phenotyping of ARDS (10). Compared to focal, patients with diffuse ARDS have lower lung compliance and higher recruitability, are more likely to respond to high PEEP and recruitment maneuvers, and are burdened by higher mortality (11).

Finally, a secondary retrospective analysis of previous trials led to the identification of two consistent ARDS subphenotypes based on a cluster of clinical and biological variables: the hypo- and the hyper-inflamed. This confirmed that the ARDS definition encompasses diseases with different natural histories, clinical and biological characteristics and outcome $(5,12)$. The two biological subphenotypes consistently showed different or even opposite responses to several treatments, with the hyper-inflated usually responding to more aggressive strategies (i.e., higher PEEP) (13).

These studies suggest that the possibility to identify biological sub-phenotypes at the bedside will provide the basis for refined personalized definition of ARDS, which is the prerequisite for correct implementation of individualized treatments (Table 1). 


\section{Personalized tidal volume}

Due to edema, alveolar flooding and increased lung weight, ARDS is characterized by reduced size of the aerated lung available for tidal ventilation: the mechanical distortion provided by $\mathrm{V}_{\mathrm{T}}$ in this "baby lung" is the most important determinant of VILI. Accordingly, lowering $\mathrm{V}_{\mathrm{T}}$ is among the most convincing interventions capable of mitigating the risk of VILI and improve clinical outcome. $V_{\mathrm{T}}$ size is conventionally scaled on predicted body weight. However, predicted body weight reflects total lung size and does not consider the extent of aeration loss and the actual lung size, that have high inter-subject variability. Therefore, some patients (i.e., those with smaller functional lung size) are at risk of hyperinflation even if $\mathrm{V}_{\mathrm{T}}$ is limited to $6 \mathrm{~mL} / \mathrm{kg}$ of predicted body weight. Since respiratory system compliance is correlated with the aerated lung size (14), the driving pressure, which is the $V_{T}$ divided by the respiratory system compliance, better reflects the effects of $\mathrm{V}_{\mathrm{T}}$ setting and represents the ventilatory variable most strongly associated to clinical outcome in ARDS (15). Current evidence supports the use of $\mathrm{V}_{\mathrm{T}}$ lower than $6 \mathrm{~mL} / \mathrm{kg}$, especially if this is associated to a driving pressure greater than $14 \mathrm{cmH}_{2} \mathrm{O}$ (16).

The occurrence of VILI depends on regional overdistension in the aerated lung: this cannot be precisely estimated by airway pressure because of the interference of chest wall elastance, which is highly inter-individually variable. Conversely, the proportion of alveolar pressure at end-inspiration that is dissipated across the lung accurately reflects the transpulmonary pressure in the non-dependent, ventilated areas. This "transpulmonary plateau pressure" is easily calculated by multiplying plateau pressure and the ratio of lung to respiratory system elastance, measured by esophageal manometry (17): limiting $\mathrm{V}_{\mathrm{T}}$ to maintain transpulmonary plateau pressure below $22-24 \mathrm{cmH}_{2} \mathrm{O}$ appears wise to prevent regional overdistension.

These considerations prompt the use of $\mathrm{V}_{\mathrm{T}}$ not exceeding $6 \mathrm{~mL} / \mathrm{kg}$ in ARDS patients; further limiting $\mathrm{V}_{\mathrm{T}}$ to on the basis of driving pressure, transpulmonary plateau pressure and/or electrical impedance tomography-derived signs of overdistension (18) appears sound but further validations are needed to identify safe thresholds. Hypercapnia can develop because of ultra-low $\mathrm{V}_{\mathrm{T}}$ strategies. Extracorporeal $\mathrm{CO}_{2}$ removal $\left(\mathrm{ECCO}_{2} \mathrm{R}\right)$ to facilitate $\mathrm{V}_{\mathrm{T}}$-reduction below $6 \mathrm{~mL} / \mathrm{kg}$ is feasible (19): further research is warranted to identify the patients that most benefit from this approach and to establish whether this strategy may improve clinical outcome.

\section{Personalized PEEP}

It is widely accepted that PEEP setting should aim to balance recruitment of collapsed lung, yielding increased aerated tissue for tidal ventilation, and the unavoidable damage caused by over-distention of already open alveoli. Five different randomized studies comparing higher versus lower PEEP, in which higher PEEP values were set according to plateau pressure (20), oxygenation impairment $(21,22)$, to generate positive transpulmonary pressure (23) and to minimize driving pressure (24) failed to detect a benefit of high PEEP strategies. Such results do not support the use of high PEEP in ARDS patients: however, the response to PEEP in terms of recruitment of previously collapse alveolar units (lung recruitability) significantly varies among subjects, and cannot be estimated by changes in the driving or transpulmonary pressure nor by improvement in oxygenation (3). High PEEP in patients with low recruitability worsens lung injury and affects hemodynamics, while low PEEP in potentially recruiting patients cannot fully exert its beneficial effects on lung protection (25). These considerations prompted a search for bedside strategies to set PEEP on the basis of patient's recruitability. The amount of recruitable lung is best investigated using computed tomography scan (3), but this technique may not be feasible in everyday clinical practice due to economical and practical issues. Recently, strategies available at the bedside based on lung volume measurement by gas dilution, electrical impedance tomography and simplified assessment of recruitment have been proposed $(25,26)$. These appear promising to aid physiology-based PEEP titration based on patient's individual response, warranting randomized trials on the topic.

\section{Personalized rescue therapies}

Prone position is the most effective way to increase lung protection and decrease mortality: its use is strongly advised in ARDS patients with $\mathrm{P} / \mathrm{F} \leq 150 \mathrm{mmHg}$ (27). Prone position recruits atelectatic lung areas, improves ventilation/perfusion matching and limits inhomogeneities, also ameliorating right ventricular function. Thus, the more these derangements are present the more effective prone position should be. Nevertheless, identifying patients who most benefit from prone position remains a clinical challenge. The improvement in oxygenation determined by prone position does not predict decreased mortality and should not be used as clinical criterion to decide to continue 
the procedure. Indeed, prone position leads to a change in the shape of the wall and the thorax, thus inducing a redistribution of $\mathrm{V}_{\mathrm{T}}$ towards the dorsal areas, thereby favoring a more homogeneous distribution of ventilation (5). As lung protection is probably the true "life-saving" effect of prone positioning, we could speculate its benefit would be maximal in subjects whose response to prone position in terms of increased lung homogeneity is more evident. However, assessing homogeneity at the bedside requires sophisticated imaging techniques (18). In the clinical scenario, arterial $\mathrm{CO}_{2}$ response to prone position, which is directly related to changes in both lung recruitment and overdistension, could be used as surrogate to confirm lung protection and guide personalized application of prone position. Hence, previous study showed that subjects improving $\mathrm{PaCO}_{2}$ have a higher survival rate at 28 days compared to non-responders. Another method to identify subjects more likely to benefit from prone position is once again to differentiate focal vs. non-focal ARDS: indeed, in focal ARDS, pronation recruits the non-aerated lung (i.e., atelectatic zones), avoiding hyperinflation of the wellventilated areas and over-distention (28).

When prone position is insufficient to determine an improvement in oxygenation and lung healing, extracorporeal membrane oxygenation (ECMO) should be considered. A recent prospective cohort study showed how ECMO could be useful in severe ARDS patients to normalize gas exchange despite ultra-protective mechanical ventilation and lung rest, potentially favoring reduction of edema and lung recovery (29). Moreover, in the recent EOLIA trial, ECMO was used as a rescue therapy in severe ARDS patients and survival improved almost significantly compared to standard protective ventilation (30); in a Bayesian analysis, ECMO was shown to be more effective than standard protective ventilation therapy in reducing mortality once physician's confidence in the effectiveness of the treatment was high, which may derive from precise recognition of sub-phenotypes more likely to respond $(31,32)$.

Less evidence is available concerning $\mathrm{ECCO}_{2} \mathrm{R}$ use in ARDS patients: while it could be useful to reduce $V_{T}$ to less than $6 \mathrm{~mL} / \mathrm{kg}$, the effect on oxygenation is nil or even negative. Indeed, $\mathrm{ECCO}_{2} \mathrm{R}$ could induce hypoxemia through multiple mechanisms including lung collapse and change in the respiratory quotient. However, at variance from $\mathrm{ECMO}$, during $\mathrm{ECCO}_{2} \mathrm{R}$ hypoxic vasoconstriction remains intact and this optimizes ventilation/perfusion matching Probably, this could support use in focal ARDS as it could improve the perfusion of the well-ventilated areas, avoiding to "wasted" blood flow through atelectasis zones. A recent analysis confirmed the possibility of personalizing use of $\mathrm{ECCO}_{2} \mathrm{R}$ and suggested that the lung-protective benefits of $\mathrm{ECCO}_{2} \mathrm{R}$ increases with lower respiratory system compliance and higher alveolar dead space fraction but not with lower $\mathrm{P} / \mathrm{F}$ ratio (33).

\section{The LIVE study: the first trial on a personalized approach to ARDS}

The recently published LIVE study was the first trial to test personalized mechanical ventilator settings in moderateto-severe ARDS (1). In the intervention group, $\mathrm{V}_{\mathrm{T}}$, PEEP, use of prone position and recruitment maneuvers were personalized according to lung morphology (i.e., focal $v s$. non-focal), which was assessed by computer tomography (CT) or chest X-ray: subjects with focal ARDS (i.e., collapse limited to the dorsal lung regions) were treated with higher $\mathrm{V}_{\mathrm{T}}$ (up to $8 \mathrm{~mL} / \mathrm{kg}$ ), lower PEEP and prone position; subjects with non-focal diffuse ARDS, instead, were managed with lower $V_{T}$, more aggressive recruitment and higher PEEP to reach upper limit of protective plateau pressure. In control group, $\mathrm{V}_{\mathrm{T}}$ was set at $6 \mathrm{~mL} / \mathrm{kg}$, low PEEP based on the degree of oxygenation impairment was delivered, and prone position use was considered a rescue strategy. Primary study outcome was mortality at 90 days and study numerosity was 420 subjects. No significant difference between the two groups was shown by univariate analysis (mortality rate was $27 \%$ vs. $27 \%$; HR 0.96 ; $95 \%$ CI, 0.66-1.4; $\mathrm{P}=0.84$ ). Secondary outcomes were not improved, either.

However, this study might have disclosed another result that could foster our knowledge on ARDS pathophysiology in unexpected directions. Indeed, experts post-hoc review of the on-site classification of focal and non-focal ARDS discovered that misclassification occurred in a high percentage of subjects ( $\mathrm{n}=85,21 \%$ of total population). When analyses were performed again considering misclassification, the 90-day mortality of the misclassified patients was higher in the personalized group [26 (65\%) of 40 patients] than in the control group [18 (32\%) of 57 patients; HR 2.8; 95\% CI, 1.5-5.1; $\mathrm{P}=0.012]$. Moreover, for the correctly classified subjects, mortality was lower in the personalized group [29 (19\%) of 156 patients] than in the control group [58 (28\%) of 204 subjects; HR 0.6; $95 \%$ CI, $0.37-0.99 ; \mathrm{P}=0.042]$. Although being secondary and lacking the adequate power, both these results could shed new light on the personalized treatment of ARDS sub-phenotypes. 
Indeed, in patients with focal ARDS, the use of high levels of PEEP and lower volumes together with recruitment maneuvers could have caused over-distention, worsening of the respiratory system compliance, increased lung stress and dead space and impaired right ventricular function. On the other hand, in non-focal ARDS, where the damage is patchy and the amount of recruitable areas is higher, lower levels of PEEP could have caused higher shunt and FiO2 requirements, while higher $\mathrm{V}_{\mathrm{T}}$ could have increased lung strain and atelectrauma. The decrease in mortality of correctly classified subjects, instead, strongly support the need of comprehensive personalized assessment of the ventilation strategy when treating a moderate-severe ARDS patient.

In the real-life of our ICUs, experts in lung morphology are not always readily available and misclassification could be even more frequent than in a specific clinical trial. Thus, clinical translation of the new findings by the LIVE study requires thoughtful understanding of the most adequate method to classify subjects. Available options could be more solid quantitative tests of recruitability or the support by artificial intelligence. For quantitative tests of recruitment, bedside dynamic imaging tools such as electrical impedance tomography or ultrasound could outperform statistic chest $\mathrm{X}$-rays and CT scans used in the LIVE study. Artificial intelligence, instead, could compare almost instantaneously the actual imaging from the patient to extremely large datasets of images pre-classified by experts. Moreover, combination of multiple indexes integrating clinical condition with physiology and biological markers could be key to fully disclose sub-phenotypes at the bedside, as ARDS is a continuum of pathophysiological patterns with blurred borders.

\section{Personalized approach at the bedside}

All the evidences discussed so far indicate that the paradigm of ARDS management shifted from correcting gas exchange to ensuring lung protection and limiting VILI (Table 1). Reduction of $\mathrm{V}_{\mathrm{T}}$ to $6 \mathrm{~mL} / \mathrm{kg} \mathrm{PBW}$ and, in moderate-tosevere patients, higher PEEP and prone positioning should be considered mandatory if no major contraindication exists (e.g., hemodynamic instability).

However, contemporary ICU physicians should also aim to implement physiological bedside measures to assess specific patient characteristics and further characterize ARDS subphenotypes. Ideally, each ventilatory setting should be accurately tailored to individual physiology. VT- reduction below $6 \mathrm{~mL} / \mathrm{kg}$ PBW should be considered if indexes of regional (measured by electrical impedance tomography) or global (measured by airway or transpulmonary elastance-derived plateau pressure) lung stress and strain (measured by airway or transpulmonary driving pressure or by dilution methods for lung aeration) exceed safety thresholds. On the other hand, $\mathrm{V}_{\mathrm{T}}$ higher than $6 \mathrm{~mL} / \mathrm{kg}$ PBW can be considered in hypercapnic ARDS patients if safety thresholds aren't crossed. Higher PEEP should be selected after thorough assessment of actual lung recruitability for which different techniques exist, based on respiratory mechanics or imaging tools. Finally, rescue therapies should be considered for more severe patients in terms of worsen oxygenation and/or more impaired lung mechanics and/or lower residual lung aeration (i.e., smaller baby lung).

\section{Conclusions}

Personalized treatment of ARDS represents the most fascinating challenge for researchers and clinicians in the next few years. Each component of treatment could be personalized after allocating patients to consistent biologically- and physiologically-sound sub-phenotypes. Given the magnitude of the required effort, caregivers at all the levels should feel involved: from funding agencies to institutions, to doctors, nurses and families, so that the focus of personalized treatment could shift from simply improve mortality to long-term functional outcomes.

\section{Acknowledgments}

The present work was funded, in part, by Departmental funding to T Mauri and E Spinelli.

\section{Footnote}

Conflicts of Interest: DL Grieco has received payments for travel expenses by Air Liquide, Maquet and Getinge, and discloses research grants by SIAARTI, ESICM and General Electric Healthcare. The other authors have no conflicts of interest to declare.

\section{References}

1. Constantin JM, Jabaudon M, Lefrant JY, et al. Personalised mechanical ventilation tailored to lung morphology versus low positive end-expiratory pressure for patients with 
acute respiratory distress syndrome in France (the LIVE study): a multicentre, single-blind, randomised controlled trial. Lancet Respir Med 2019;7:870-80.

2. ARDS Definition Task Force, Ranieri VM, Rubenfeld GD, et al. Acute respiratory distress syndrome: the Berlin Definition. JAMA 2012;307:2526-33.

3. Gattinoni L, Caironi P, Cressoni M, et al. Lung recruitment in patients with the acute respiratory distress syndrome. N Engl J Med 2006;354:1775-86.

4. Acute Respiratory Distress Syndrome Network, Brower RG, Matthay MA, et al. Ventilation with lower tidal volumes as compared with traditional tidal volumes for acute lung injury and the acute respiratory distress syndrome. N Engl J Med 2000;342:1301-8.

5. Gattinoni L, Taccone P, Carlesso E, et al. Prone position in acute respiratory distress syndrome. Rationale, indications, and limits. Am J Respir Crit Care Med 2013;188:1286-93.

6. Gattinoni L, Marini JJ, Pesenti A, et al. The "baby lung" became an adult. Intensive Care Med 2016;42:663-73.

7. Mauri T, Yoshida T, Bellani G, et al. Esophageal and transpulmonary pressure in the clinical setting: meaning, usefulness and perspectives. Intensive Care Med 2016;42:1360-73.

8. Coppola S, Froio S, Marino A, et al. Respiratory Mechanics, Lung Recruitability, and Gas Exchange in Pulmonary and Extrapulmonary Acute Respiratory Distress Syndrome. Crit Care Med 2019;47:792-9.

9. Maiolo G, Collino F, Vasques F, et al. Reclassifying Acute Respiratory Distress Syndrome. Am J Respir Crit Care Med 2018;197:1586-95.

10. Bellani G, Mauri T, Pesenti A. Imaging in acute lung injury and acute respiratory distress syndrome. Curr Opin Crit Care 2012;18:29-34.

11. Constantin JM, Grasso S, Chanques G, et al. Lung morphology predicts response to recruitment maneuver in patients with acute respiratory distress syndrome. Crit Care Med 2010;38:1108-17.

12. Mauri T, Coppadoro A, Bellani G, et al. Pentraxin 3 in acute respiratory distress syndrome: an early marker of severity. Crit Care Med 2008;36:2302-8.

13. Spadaro S, Park M, Turrini C, et al. Biomarkers for Acute Respiratory Distress syndrome and prospects for personalised medicine. J Inflamm (Lond) 2019;16:1.

14. Grieco DL, Russo A, Romanò B, et al. Lung volumes, respiratory mechanics and dynamic strain during general anaesthesia. Br J Anaesth 2018;121:1156-65.

15. Amato MBP, Meade MO, Slutsky AS, et al. Driving pressure and survival in the acute respiratory distress syndrome. N Engl J Med 2015;372:747-55.

16. Grieco DL, Chen L, Dres M, et al. Should we use driving pressure to set tidal volume? Curr Opin Crit Care 2017;23:38-44.

17. Yoshida T, Amato MBP, Grieco DL, et al. Esophageal Manometry and Regional Transpulmonary Pressure in Lung Injury. Am J Respir Crit Care Med 2018;197:1018-26.

18. Frerichs I, Amato MBP, van Kaam AH, et al. Chest electrical impedance tomography examination, data analysis, terminology, clinical use and recommendations: consensus statement of the TRanslational EIT developmeNt stuDy group. Thorax 2017;72:83-93.

19. Combes A, Fanelli V, Pham T, et al. Feasibility and safety of extracorporeal $\mathrm{CO} 2$ removal to enhance protective ventilation in acute respiratory distress syndrome: the SUPERNOVA study. Intensive Care Med 2019;45:592-600.

20. Mercat A, Richard JM, Vielle B, et al. Positive endexpiratory pressure setting in adults with acute lung injury and acute respiratory distress syndrome: a randomized controlled trial. JAMA 2008;299:646-55.

21. Brower RG, Lanken PN, MacIntyre N, et al. Higher versus lower positive end-expiratory pressures in patients with the acute respiratory distress syndrome. N Engl J Med 2004;351:327-36.

22. Meade MO, Cook DJ, Guyatt GH, et al. Ventilation strategy using low tidal volumes, recruitment maneuvers, and high positive end-expiratory pressure for acute lung injury and acute respiratory distress syndrome: a randomized controlled trial. JAMA 2008;299:637-45.

23. Beitler JR, Sarge T, Banner-Goodspeed VM, et al. Effect of Titrating Positive End-Expiratory Pressure (PEEP) With an Esophageal Pressure-Guided Strategy vs an Empirical High PEEP-Fio2 Strategy on Death and Days Free From Mechanical Ventilation Among Patients With Acute Respiratory Distress Syndrome: A Randomized Clinical Trial. JAMA 2019;321:846-57.

24. Writing Group for the Alveolar Recruitment for Acute Respiratory Distress Syndrome Trial (ART) Investigators, Cavalcanti AB, Suzumura ÉA, et al. Effect of Lung Recruitment and Titrated Positive EndExpiratory Pressure (PEEP) vs Low PEEP on Mortality in Patients With Acute Respiratory Distress Syndrome: A Randomized Clinical Trial. JAMA 2017;318:1335-45.

25. Chen L, Del Sorbo L, Grieco DL, et al. Potential for Lung Recruitment Estimated by the Recruitment-toInflation Ratio in Acute Respiratory Distress Syndrome. 
Am J Respir Crit Care Med 2019. [Epub ahead of print].

26. Mauri T, Eronia N, Turrini C, et al. Bedside assessment of the effects of positive end-expiratory pressure on lung inflation and recruitment by the helium dilution technique and electrical impedance tomography. Intensive Care Med 2016;42:1576-87.

27. Guérin C, Reignier J, Richard JC, et al. Prone positioning in severe acute respiratory distress syndrome. $\mathrm{N}$ Engl J Med 2013;368:2159-68.

28. Mauri T, Grasselli G, Pesenti A. Systematic assessment of advanced respiratory physiology: precision medicine entering real-life ICU? Crit Care 2017;21:143.

29. Schmidt M, Pham T, Arcadipane A, et al. Mechanical Ventilation Management during Extracorporeal Membrane Oxygenation for Acute Respiratory Distress Syndrome. An International Multicenter Prospective Cohort. Am J Respir Crit Care Med 2019;200:1002-12.

Cite this article as: Spinelli E, Grieco DL, Mauri T. A personalized approach to the acute respiratory distress syndrome: recent advances and future challenges. J Thorac Dis 2019;11(12):5619-5625. doi: 10.21037/jtd.2019.11.61
30. Combes A, Hajage D, Capellier G, et al. Extracorporeal Membrane Oxygenation for Severe Acute Respiratory Distress Syndrome. N Engl J Med 2018;378:1965-75.

31. Goligher EC, Tomlinson G, Hajage D, et al. Extracorporeal Membrane Oxygenation for Severe Acute Respiratory Distress Syndrome and Posterior Probability of Mortality Benefit in a Post Hoc Bayesian Analysis of a Randomized Clinical Trial. JAMA 2018;320:2251-9.

32. Spinelli E, Mauri T, Carlesso E, et al. Time-Course of Physiologic Variables During Extracorporeal Membrane Oxygenation and Outcome of Severe Acute Respiratory Distress Syndrome. ASAIO J 2019. doi: 10.1097/ MAT.0000000000001048.

33. Gattinoni L, Vassalli F, Romitti F, et al. Extracorporeal gas exchange: when to start and how to end? Crit Care 2019;23:203. 